\title{
SAND99-1504C
}

\section{Large Distributed Data Acquisition System at the Z Facility*}

\author{
Jerry A. Mills \\ Ktech Corporation \\ 2201 Buena Vista SE, Ste 400 \\ Albuquerque, NM 87106 \\ James E. Potter \\ Sandia National Laboratories \\ PO Box 5800 MS 1192 \\ Albuquerque, NM 87185
}

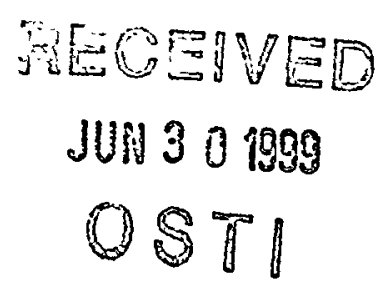

\section{Abstract}

Experiments at the $\mathrm{Z}$ machine generate over four hundred channels of waveform data on each accelerator shot. Most experiments require timing accuracy to better than one nanosecond between multiple distributed recording locations throughout the facility. Experimental diagnostics and high speed data recording equipment are typically located within a few meters of the 200 to 300 terawatt $X$ - ray source produced during Z-pinch experiments. This paper will discuss techniques used to resolve the timing of the several hundred data channels acquired on each shot event and system features which allow viewing of waveforms within a few minutes after a shot. Methods for acquiring high bandwidth signals in a severe noise environment will also be discussed.

\section{Introduction}

The $Z$ Facility is a 36 module accelerator system comprised of Marx Generators, water Storage capacitors, LASER triggered gas switches, and pulse forming lines that feed Magnetically Insulated Transmission Lines (MITLs) contained in a ten foot diameter vacuum chamber. Voltage and current measurements are made on each of these subsystems on each shot as well as X-ray diagnostics that are located at the end of Line-of-Sight (LOS) pipes. Timing between the digitizer locations must be maintained to better than one nanosecond to allow accurate representation of machine operation and X-ray output for analysis.

\section{System Information}

The waveform recording section of the Data Acquisition System (DAS) at the $Z$ Facility currently consists of 14 Tektronix TLS216 Logic Analyzers, 12 Analytek recorders, 9 Tektronix TDS640 Digitizing Oscilloscopes, 5 Tektronix TDS684 Digitizing Oscilloscopes, 26 Tektronix TVS645 VXI Waveform Recorders and associated cable plant and signal conditioning equipment for fast transient signals. An additional 5 LeCroy 8210 modules allow for recording of slower signals. Digitizers are controlled via fiber-optically coupled GPIB connections on an HP9000 K-Class server

using ethernet addressing. This equates to more than 450 waveform channels available on any given test event. Figure 1 shows a block diagram of the $Z$ DAS recording and timing systems.

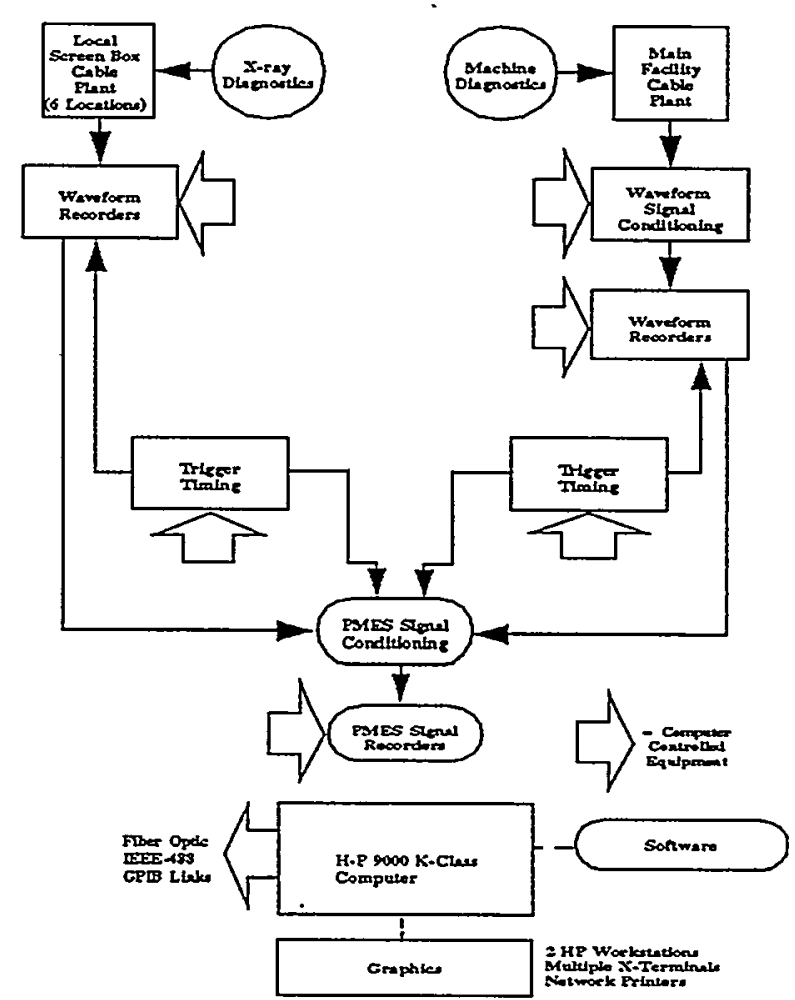

Figure 1: Z Data Acquisition System Block Diagram

The DAS also uses a Performance Monitoring and Evaluation System (PMES) to record single point timing information. This system is comprised of 14 LeCroy 4208 Time-to-Digital Converter (TDC) modules sampling at $1 \mathrm{~ns}$ for $+1-7 \mathrm{~ms}$ and 12 Berkeley Nucleonics B980 TDC modules sampling at 48ps for 13 seconds. This provides capability to record 208 channels of timing data. BNC980 timers are currently used only for recorder trigger-in/out timing to obtain the most accurate waveform timing possible. 


\section{DISCLAIMER}

This report was prepared as an account of work sponsored by an agency of the United States Government. Neither the United States Government nor any agency thereof, nor any of their employees, make any warranty, express or implied, or assumes any legal liability or responsibility for the accuracy, completeness, or usefulness of any information, apparatus, product, or process disclosed, or represents that its use would not infringe privately owned rights. Reference herein to any specific commercial product, process, or service by trade name, trademark, manufacturer, or otherwise does not necessarily constitute or imply its endorsement, recommendation, or favoring by the United States Government or any agency thereof. The views and opinions of authors expressed herein do not necessarily state or reflect those of the United States Government or any agency thereof. 


\section{DISCLAIMER}

Portions of this document may be illegible in electronic image products. Images are produced from the best available original document. 


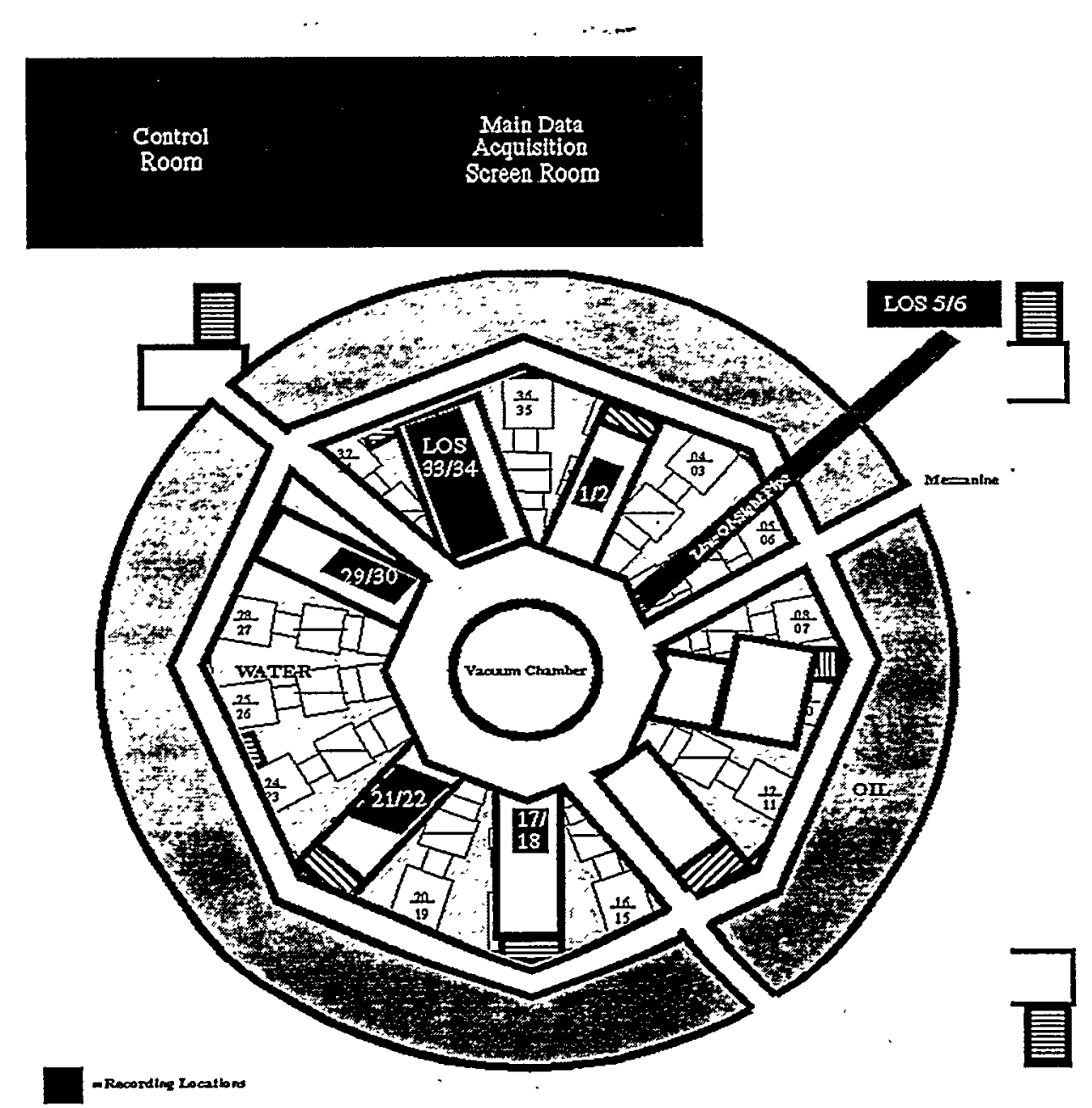

Figure 2: DAS Recording Locations

\section{System Layout}

Digitizers are located throughout the facility as seen in Figure 2.

The digitizers for $\mathrm{X}$-ray information are located in Double-wall Faraday Cages on platforms arranged like spokes around the central vacuum chamber. The location of these screen boxes take advantage of low signal loss for fast rise signals due to proximity to $\mathrm{X}$-ray source diagnostics and the use of short runs of high quality coaxial cable. X-ray information is primarily recorded using the TDS684 and TVS645 units with a sample rate of 200ps/sample.

Machine diagnostics are recorded on TDS640, TLS216, and Analytek units located in a large Doublewall Faraday Cage located somewhat further away from the machine. Signal routing takes place on significantly longer cable runs than are used for X-ray diagnostics. These signals do not have the fast rise times seen on $\mathrm{X}$ ray diagnostic signals and can have the frequency loss of the cable run added back by characterization of the run and the use of system software. Sample rates of $500 \mathrm{ps} / \mathrm{sample}$ to $2 \mathrm{~ns} /$ sample are typical for these signals.
The PMES system is also located in this large screen room

$\therefore$

$\therefore \quad$ All digitizers used are extensively characterized to determine trigger-in to trace start time and/or trigger-in to trigger-out throughput timing. Each digitizer system sends at least a delay generator output signal and either a digitizer trigger-in or trigger-out to the PMES, which is the heart of timing information at the facility. DAS software setups take into account each cable that a signal passes through on its way to the recorder. These lengths are typically measured to better than $+1-50$ ps using either TDR measurements or Time Interval Meter measurements made using a Stanford Research Systems SR620 and can include obtaining more than one measurement depending on signal conditioning requirements.

All timing ultimately ties to $\mathrm{CM} 1$ or $\mathrm{A} 0$ trigger time. CM1 is the trigger source of the TG100, which is the initial trigger to nine Mini-Marx Generators that make up the MTG subsystem, which in turn trigger the 36 Marx Generators. DAS software again takes into account cable lengths of the digitizer signal to PMES and performs 
addition and subtraction of signals to obtain precise timing to a common timing point (A $\overline{0}$ ).

Signal timing is determined by entries into the DAS downline header and a separate PMES header that manipulate the data to create normalized data arrays. The DAS downline header uses three of twenty-one columns as a basis for calculating timing. The first column used is labeled 'DASCAB' and contains cable lengths from either the main Screen Room patch panel or the feedthroughs on a remote Screen Box entered in nanoseconds. The second column is an entry for length of cable from the main screen room to the diagnostic monitor for machine diagnostics, or as the Time-of-Flight for X-rays to travel up a Line-of-Sight pipe when recording $X$-ray diagnostics. This column is labeled either 'HIBAY' or 'TOF'. These also are entered in nanoseconds. Waveforms are shifted negative in time for both of these values.

The third column used begins to make use of PMES data acquired. Each digitizer has an alpha-numeric entry referring to a line in the PMES header. This PMES line does several mathematical operations on the timing data that is then automatically applied to the signal prior to being written as a normalized data array. An example of a downline header entry:

C sticon Diale Signats . SCREEN BOX 12 . TVBGAS

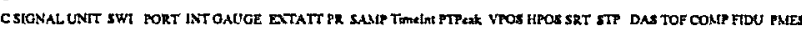

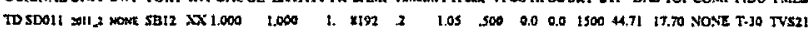

The PMES line first subtracts out the length of cable from the digitizer trigger output, or Trigger-Input in the case of TVS645 units, to the PMES Input. The next step is for the PMES header line to add the time stored in the TDC to the time of a separate PMES channel. This channel records the Trigger Delay arrival time at a fanout which is providing triggers to all digitizers in one location. This signal is split as it arrives at PMES and provides the start pulse for the TDC that is referenced in the downline header. This signal does not use any correction for cable lengths.

The final step is the subtraction of the sum from the downline header reference from $\mathrm{A} 0$, which is listed in the PMES header as 'REFl'. Data is then shifted positively by the value calculated in the PMES software routine. An example of PMES lines required to complete timing calculations:

C TIMING REFERENCE SIGNALS

DEV SIGNAL MODEL \#CHLAN CHAN!

II REF1 4208 I 57 CO

CI LINE-\# DASCAB SIGCON ACTMMI ACTMM2 ADDSIG LINE-\# SUBSIG LINE-\#\#

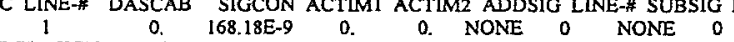
DEV SIGNAL MODEL \#CHAN CHAN

II REFSBI2 980 i $214 \mathrm{CO}$

C LINE-\# DASCAB SIGCON ACTIM1 ACTIM2 ADDSIG LINE-\# SUBSIG LINE-\#\# $\begin{array}{llllllll}1 & 0.00 \mathrm{E}-9 & 0.0 \mathrm{E}-9 & 0.0 \mathrm{E}-9 & 0.0 \mathrm{E}-9 & \mathrm{NONE} & 0 & \underline{R E F 1}\end{array}$

C

DEV SIGNAL MODEL \#CHAN CHANI

TI TVS211 980 I 265 CO

C LINE-\# DASCAB SIGCON ACTIM1 ACTIM2 ADDSIG LINE-\# SUBSIGLINE-H\#

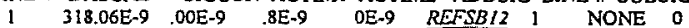

\section{Timing Verification}

Timing verification takes place using a single fast risetime pulse that is split to each recording location and routed on precisely measured cables. This signal is currently recorded typically on the first channel of a digitizer on which a signal of interest also is recorded. This pulse is referred to as the Common Reference signal and may be split to other recorders at a location if required to provide cross checks of timing within one location. Spreads on this signal are typically $+/-300$ ps on any given shot for 15 separate recorders. The Common Reference signals provide a means of manually determining absolute timing of similar signals at widely spaced recording locations. Figure 3 shows the Common Reference results from a typical shot.

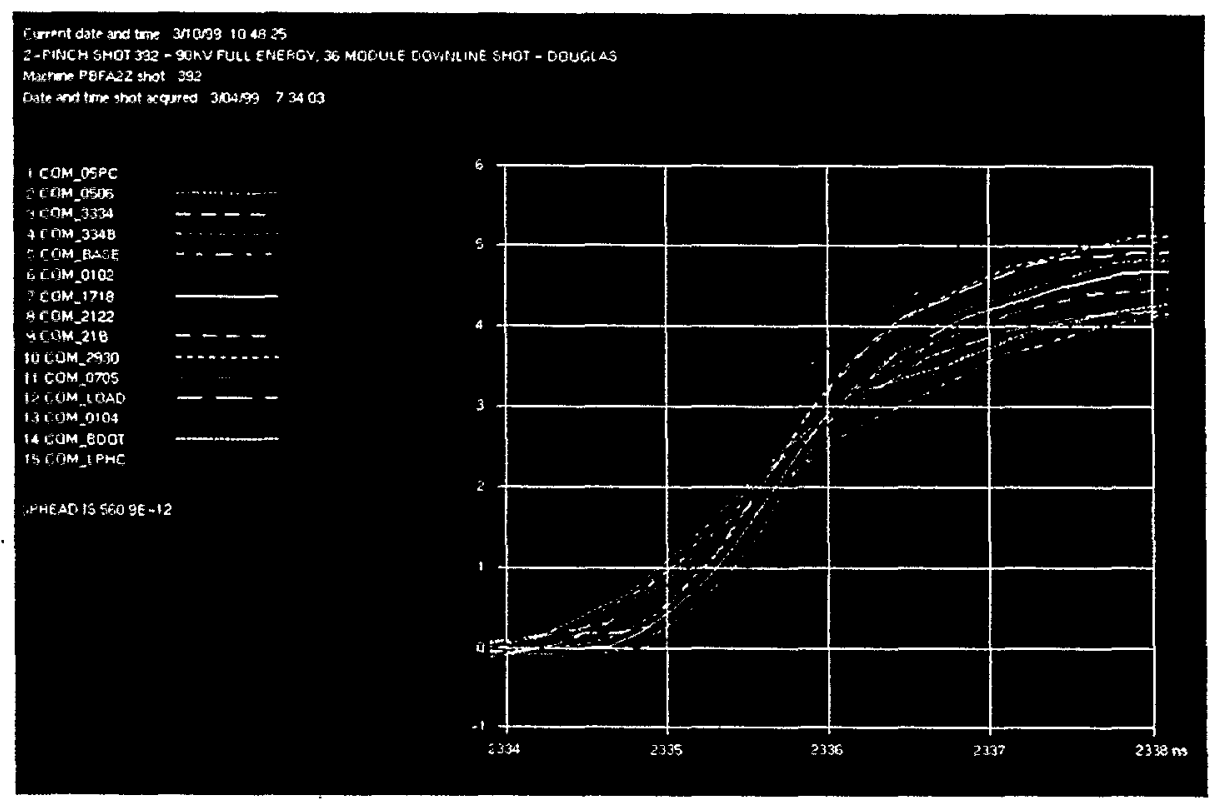

Figure 3: Common Reference Signal Timing 


\section{Rapid Access to Normalized Data}

Using features of the software routines used to acquire data at the $Z$ facility, normalized PMES and waveform recorder data, which have been corrected for amplitude and time, start becoming available within three minutes after the machine fires. PMES system signals are read first and are followed by X-ray diagnostic signals. Each recorder is read and processed individually with signals being read in any order the Experimenter wishes. Machine diagnostic data is typically read and processed last. Over 400 channels are read and processed completely in approximately 15 minutes

\section{Special Techniques for Acquiring Data near the X-ray Source}

Digitizers located in screen boxes near the $\mathrm{X}$-ray source are also situated in a high Electro-Magnetic Pulse (EMP) environment. The measurements recorded here are very fast risetime and require short cable runs on high quality coaxial cables. The cables used are typically 22 feet of Andrew LDF4-50A 1/2" Heliax cables using ' $N$ ' Type connectors that run from the diagnostic output to the outer shell of the Screen Box. Inside the screen box is an eight foot run of RG9914. Special care is taken to minimize noise on the cable runs by installing an outer steel braid to the Heliax cable runs and securing one end to the ' $N$ ' connector at the diagnostic and attaching the opposite end to the Screen Box feedthrough. The connector at the screen box feedthrough is isolated electrically by wraps of electrical tape. The braid is then mechanically attached to the feedthrough housing and thus basically creates a triaxial cable run. The combination of Heliax and RG9914 cables creates a cable run to allow signals recording in excess of $4 \mathrm{GHz}$, which exceeds the digitizer recording capability. Figure 4 shows details of screen box connections.

\section{Summary}

It takes a great deal of work but sub-nanosecond resolution of data timing is possible in a large data acquisition system. Noise levels can be kept to a minimum even in an extreme electrical noise environment with careful thought and execution of component installation. Work on improvements to our system continue. Replacing Analytek digitizers with TektronixTLS216 recorders and elimination of the remaining LeCroy 4208 timers by use of BNC B980 TDCs will only increase our timing accuracy. The search for faster recorders is an ongoing endeavor and the Z DAS will continue to pursue the most up-to-date recording and timing systems available.

\section{Acknowledgments}

Special thanks to John McGurn for his help in characterizing the fast data cable runs and establishing the Common Reference system for timing verification.

Grateful appreciation to Jeff Slopek and Tom Dinwoodie for their tireless efforts in measuring and re- measuring of cable runs, as well as assistance in characterization of the DAS timing system components.

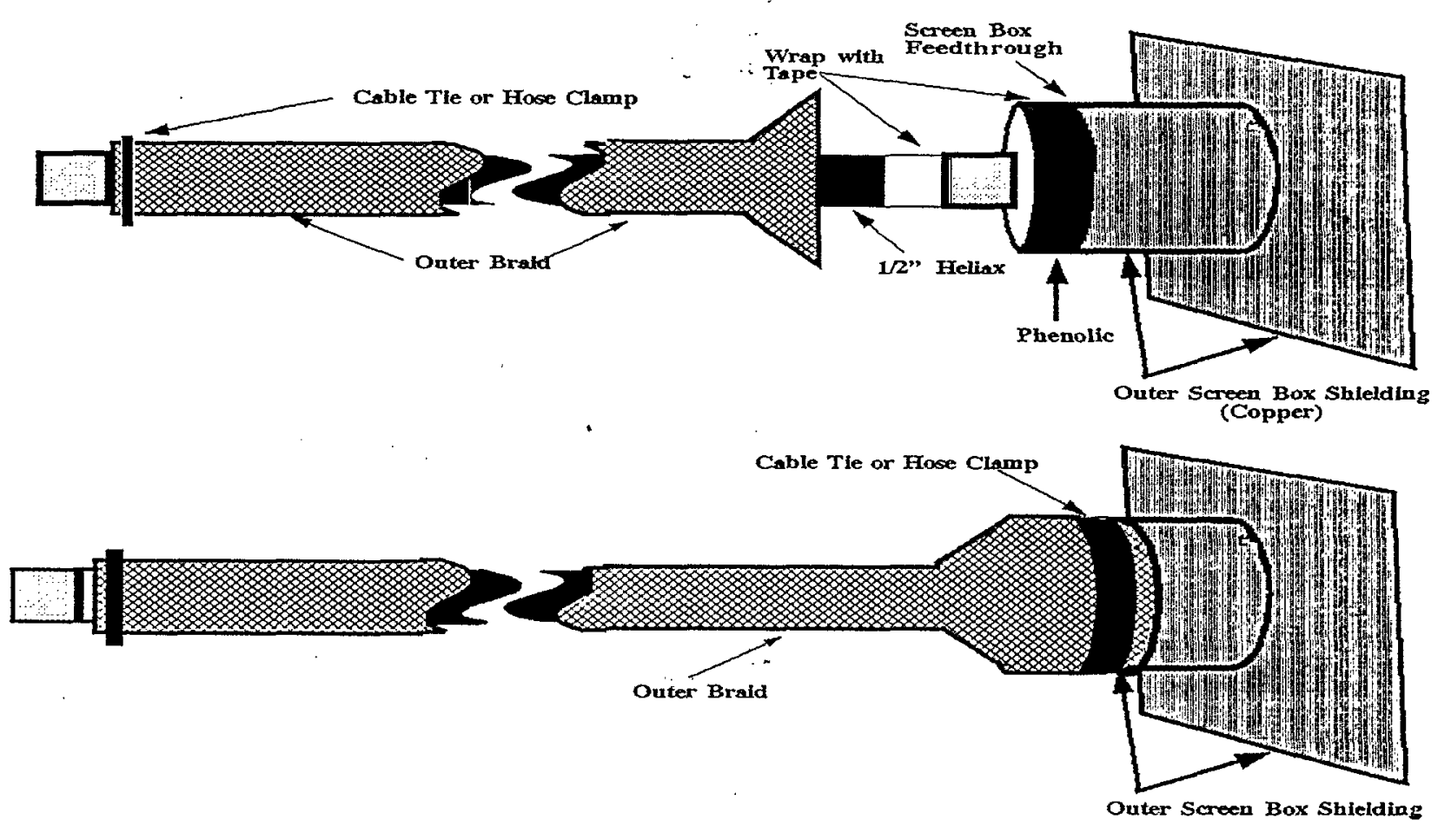

Figure 4: Screen Box Connections 